\title{
Dynamical Systems on Quantum Tori Lie Algebras
}

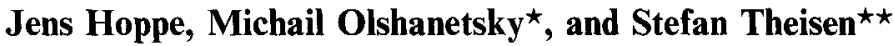 \\ Institut für Theoretische Physik, Universität Karlsruhe, Kaiserstr. 12, \\ D-76131 Karlsruhe, Germany
}

Received December 20, 1991; in revised form October 30, 1992

\begin{abstract}
We use quantum tori Lie algebras (QTLA), which are a one-parameter family of sub-algebras of $g l_{\infty}$, to describe local and non-local versions of the Toda systems. It turns out that the central charge of QTLA is responsible for the non-locality. There are two regimes in the local systems - conformal for irrational values of the parameter and non-conformal and integrable for its rational values. We also consider infinite-dimensional analogs of rigid tops. Some of these systems give rise to "quantized" (magneto-)hydrodynamic equations of an ideal fluid on a torus. We also consider infinite dimensional versions of the integrable Euler and Clebsch cases.
\end{abstract}

\section{Introduction}

Infinite dimensional Lie algebras are natural to describe symmetries of integrable systems in $1+1$ and $2+1$ dimensions. For example, Kac-Moody algebras are known to describe symmetries in $1+1$ dimensions (cf., for example [1]). $g l_{\infty}$ and its subalgebras arise in the case of $2+1$ dimensions; see [2, 3] for KP and [4] for the two-dimensional infinite Toda chain. Some new systems were considered in [5-7]. The Virasoro algebra plays a role in the description of the symmetries of the $\mathrm{KdV}$ equation [8] and some other equations [9].

Here we consider trigonometrical Lie algebras $\mathscr{L}_{A}[10]$, which, having Connes non-commutative geometry [11] in mind, are also called quantum tori Lie algebras (QTLA). This is due to the fact that they arise as the natural commutator of associative algebras $\mathscr{A}_{A}$ that are generated by two non-commutative elements $U_{1}$ and $U_{2}$ satisfying $U_{1} U_{2}=e^{4 \pi i \Lambda} U_{2} U_{1}$ [12]. The algebras are also related to $S U(\infty)$ Yang-Mills theories and membranes [13-15] and are possible candidates

\footnotetext{
* Permanent address: Institute for Theoretical and Experimental Physics, 117258 Moscow, Russia




for the universal algebra in the description of all integrable interactions which arise in string theory [16].

QTLA's can be considered as a one parameter $(\Lambda)$ family of subalgebras of $g l_{\infty}$, the Lie algebra of all two-sided infinite dimensional matrices with only a finite number of non-zero diagonals. They can be characterized as matrices with (quasi)periodic dependence along the diagonals for (ir)rational values of $\Lambda$.

Starting from a Lie algebra one can naively try to derive dynamical systems with additional integrals of motion. It is then not guaranteed that the resulting systems are completely integrable. There exists however the so-called AdlerKostant-Symes scheme, based on Hamiltonian reduction, which does yield integrable systems. This approach was developed and extended by the Leningrad school; cf. $[17,18]$ and references therein. It allows, starting from a Lie algebra and an additional structure related to the classical $r$-matrix, to derive the Lax representation, conservation laws, classical solutions to the corresponding Riemann problem and so on. All steps to be performed are more or less straightforward, following the recipe laid out in [17]. Therefore we will use their approach only for constructing the Lax pairs for integrable systems based on QTLA's.

Our interest in the systems under consideration is based on the idea that algebras that from the very beginning play some role in physical systems often lead to non-trivial dynamical systems, which eventually lead to physical applications. It is worthwhile to emphasize that these algebras describe hidden symmetries of the resulting systems.

From this point of view QTLA are good candidates. The simplest system that we obtain in the above way is the well-known Michailov-Ueno-Takasaki Toda chain [4] which is a universal object in the theory of integrable systems. As such it appeared recently also in the matrix approach to two-dimensional gravity. This is one more reason why we feel that QTLA's deserve thorough investigation within this approach. In a similar fashion we also derive the non-local version of the Toda system and find that the central charge of the QTLA is responsible for the non-locality.

In the Toda systems we find that their properties depend crucially on the parameter $A$, leading to conformal theories for $A$ rational and integrable theories otherwise. It is also easy to generalize our construction to the whole Toda hierarchy and to describe the dressing procedure for it in the spirit of [4]. Tops for finite dimensional Lie algebras and for the algebra of symplectic diffeomorphisms were introduced by Arnold [19]. There is a lot of work devoted to generalized tops on finite-dimensional algebras (cf. $[17,20]$ and references therein). The Euler top on the Virasoro algebra, which is in fact the $\mathrm{KdV}$ equation, was considered in $[21,9]$. Top-like systems naturally arise for the trigonometric algebra because in the limit $\Lambda \rightarrow 0$ it coincides with the algebra of symplectic diffeomorphisms on a torus. In this case the Euler top is equivalent to the hydrodynamics of an ideal fluid on a torus (this is a particular case of the Arnold approach) and a top in a gravitational field to magneto-hydrodynamics of a superconducting ideal fluid. Thus the systems under consideration correspond to "quantum" versions of these equations where $A$ plays the role of Planck's constant.

The paper is organized as follows. In Sect. 2 we present some preliminaries (to be used in subsequent sections) which contain two types of results. We first describe our main object, QTLA and three realisations which we will use to construct dynamical equations. We then present some $\mathbf{Z}_{2}$ gradations of QTLA and finally its coadjoint action. The former part is needed for deriving the equations for the 
top-like systems whereas the latter leads directly to a generic form of equations related to the Lax or Zacharov-Shabat equations. We also include here a brief discussion of the Leningrad version of the Adler-Kostant-Symes scheme [17] which also serves as a justification for our choice of Lax equations. After these preliminaries we consider in Sect. 3 several dynamical systems, starting with Toda-like systems. We then treat generalized Euler equations on QTLA and their integrable versions. We end with a discussion of generalized tops in an external field, magneto-hydrodynamics and its integrable version.

\section{Generalities}

2.1. Quantum Tori Algebras, $g l_{\infty}$, Representations, and Subalgebras. Consider the non-commutative associative algebra $\mathscr{A}_{\mathcal{A}}$ over $\mathbb{C}$, generated by two elements $\left\{U_{1}, U_{2}\right\}$,

$$
U_{1} U_{2}=\omega U_{2} U_{1},
$$

where $\omega=e^{4 \pi i \Lambda}$. Thus $\mathscr{A}_{\Lambda}=\left\{c_{m n} U_{1}^{m} U_{2}^{n}\right\}=\left\{\sum_{\vec{m}} \tilde{c}_{\vec{m}} T_{\vec{m}}\right\}\left(\vec{m}=\left(m_{1}, m_{2}\right) \in \mathbb{Z}\right)$ with $T_{\bar{m}}=\frac{i}{4 \pi \Lambda} \omega^{\frac{1}{2} m_{1} m_{2}} U_{1}^{m_{1}} U_{2}^{m_{2}}$. This describes a non-commutative (or quantum) twotorus [11]. The following three representations of $\mathscr{A}_{\Lambda}(\Lambda$ irrational), given in refs. $[22,23,13]$, respectively, will be used below:

$$
\begin{aligned}
& T_{\vec{m}}=\frac{i}{\lambda} \omega^{\frac{m_{1} m_{2}}{2}} e^{i m_{1} \theta} e^{m_{2} \lambda \partial_{\theta}}=\frac{i}{\lambda} e^{i m_{1} \theta+m_{2} \lambda \partial_{\theta}}, \\
& T_{\vec{m}}=\frac{i}{\lambda} \omega^{\frac{m_{1} m_{2}}{2}} \sum_{k \in \mathbb{Z}} \omega^{m_{1} k} E_{k, k+m_{2}}, \\
& T_{\vec{m}}=\frac{i}{\lambda} e^{i \vec{m} \cdot \vec{\varphi}} .
\end{aligned}
$$

We have defined

$$
\lambda=4 \pi \Lambda, \quad \omega=e^{i \lambda} .
$$

In all three representations we have

$$
T_{\vec{m}} T_{\vec{n}}=\frac{i}{\lambda} \omega^{-\frac{1}{2} \vec{m} \times \vec{n}} T_{\vec{m}+\vec{n}}
$$

Multiplication in representations (2.4) is via the star product (cf. below). In the following we will refer to (2.2) and (2.4) as vertex and star product representations, respectively. Representation (2.3) is the embedding into $g l_{\infty}$, i.e. the algebra of all two-sided infinite dimensional matrices with only a finite number of non-zero diagonals. $E_{i j}$ denotes the infinite-dimensional matrix with value 1 at position $(i, j)$ and 0 everywhere else (i.e. $\left.\left(E_{i j}\right)_{k l}=\delta_{i k} \delta_{j l}\right)$ obeying

$$
\left[E_{i j}, E_{k l}\right]=\delta_{j k} E_{i l}-\delta_{i l} E_{k j}, \quad i, j, k, l \in \mathbb{Z} .
$$

Note that $T_{\vec{m}}$ in (2.3) is zero everywhere except on the $m_{2}{ }^{\text {th }}$ off-diagonal, on which all elements are different from zero (in fact, up to the factor $\frac{i}{\lambda}$, they are powers of $\omega$, 
i.e. have absolute value one). Representation (2.2), or, equivalently ${ }^{1}$

$$
T_{\vec{m}}=-\frac{i}{\lambda} e^{i m_{2} \theta+m_{1} \lambda \partial \theta}
$$

should be thought of as acting on smooth periodic functions of $\theta$. If we write for a general element $F=\sum_{\vec{m}} f_{\vec{m}} T_{\vec{m}}$ of $\mathscr{L}_{\boldsymbol{A}}$ in the vertex representation

$$
F=\sum_{m} f_{m}(\theta) T^{m},
$$

where $T=e^{\lambda \partial_{\theta}}$ is the shift operator which satisfies

$$
T^{m} f(\theta)=f(\theta+\lambda m) T^{m},
$$

this becomes, expressed in the representation (2.3)

$$
F=\sum_{m \in \mathbb{Z}} \sum_{k \in \mathbb{Z}} f_{m}(4 \pi k \Lambda) E_{k, k+m}
$$

We have thus the embedding of $f_{m}(\theta) T^{m}$ in $g l_{\infty}$ as an $m$-off-diagonal matrix with quasi-periodic dependence along the non-vanishing diagonal:

$$
f_{m}(\theta) T^{m} \rightarrow \operatorname{diag}_{m}\left(\ldots, f_{m}(-4 \pi \Lambda), f_{m}(0), f_{m}(4 \pi \Lambda), \ldots\right) .
$$

This will be relevant when comparing our Lax operators for the local Toda system in Sect. 3.1 with the ones of [4].

Finally, the (associative) "star-product" $\star$ in (2.4) is defined for any pair $(f, g)$ of smooth functions on the torus as

$$
(f \star g)(\vec{\varphi}):=f g+\sum_{n=1}^{\infty} \frac{\left(\frac{i \lambda}{2}\right)^{n}}{n !} \varepsilon_{r_{1} s_{1}} \ldots \varepsilon_{r_{n} s_{n}}\left(\partial_{r_{1} \ldots r_{n}}^{n} f\right)\left(\partial_{s_{1} \ldots s_{n}}^{n} g\right)
$$

such that

$$
e^{i \vec{m} \cdot \vec{\varphi}} \star e^{i \vec{n} \cdot \vec{\varphi}}=\omega^{-\frac{1}{2} \vec{m} \times \vec{n}} e^{i(\vec{m}+\vec{n}) \cdot \vec{\varphi}} ;
$$

(cf. (2.6)). The $\star$-product may also be defined by the formula

$$
(f \star g)(\vec{\varphi}):=-\frac{i}{\pi^{2} \lambda^{3}} \int d \vec{\varphi}^{\prime} d \vec{\varphi}^{\prime \prime} e^{\frac{2 i}{\lambda}\left|\vec{\varphi}^{\prime} \vec{\varphi}^{\prime \prime}\right|} f\left(\vec{\varphi}^{\prime}\right) g\left(\vec{\varphi}^{\prime \prime}\right)
$$

with

$$
\left|\vec{\varphi} \vec{\varphi}^{\prime} \vec{\varphi}^{\prime \prime}\right|:=\vec{\varphi} \times \vec{\varphi}^{\prime}+\vec{\varphi}^{\prime} \times \vec{\varphi}^{\prime \prime}+\vec{\varphi}^{\prime \prime} \times \vec{\varphi}
$$

This definition extends to the case where $f$ and $g$ are integrable, but not necessarily smooth.

When working with the $\star$-product representation it is useful to note that

$$
\int \frac{d \vec{\varphi}}{(2 \pi)^{2}} f \star g=\int \frac{d \vec{\varphi}}{(2 \pi)^{2}} f g=: \operatorname{Tr}(f g) .
$$

${ }^{1}$ Concerning the Lie algebra, any $T_{\vec{m}}= \pm \frac{i}{\lambda} e^{i \vec{m} \cdot \vec{A}}$ will do as long as $\left[A_{1}, A_{2}\right]= \pm i \lambda 1$ 
Thus, we may take (2.15) as an invariant trace on the associative algebra. In the representation (2.2), an invariant trace is (cf. (2.8))

$$
\operatorname{Tr}\left(\sum_{m \in \mathbb{Z}} f_{m}(\theta) e^{m \lambda \lambda \theta_{\theta}}\right):=\int f_{0}(\theta) \frac{d \theta}{2 \pi}=\int \frac{d \theta}{2 \pi} \frac{d T}{T} F(\theta, T) .
$$

We now define $\mathscr{L}_{\Lambda}$ as the associated Lie-algebra constructed from $\mathscr{A}_{A}$ by the standard commutator. $\mathscr{L}_{A}$ consists of all finite linear combinations (over $\mathbb{C}$ ) of basis elements $T_{\overrightarrow{\mathrm{m}}}$ with commutation relations

$$
\left[T_{\vec{m}}, T_{\grave{n}}\right]=\frac{1}{2 \pi \Lambda} \sin (2 \pi \Lambda(\vec{m} \times \vec{n})) T_{\vec{m}+\vec{n}} \quad \Lambda \in(0,1) .
$$

This is the class of trigonometric Lie algebras introduced in [10]. With reference to our discussion of $\mathscr{A}_{A}$, we will call them Quantum Tori Lie Algebras (QTLA). The invariant trace and the two-coycle (cf. below) on $\mathscr{L}_{A}$ correspond, in the language of [11] to the canonical trace $\tau\left(\sum_{\vec{m}} c_{\vec{m}} T_{\vec{m}}\right)=c_{\overrightarrow{0}}$ and an element of the first cyclic cohomology group, respectively.

The $\Lambda \rightarrow 0$ limit $\mathscr{L}_{0}$ is analogously defined by

$$
\left[T_{\vec{m}}, T_{\bar{n}}\right]=(\vec{m} \times \vec{n}) T_{\vec{m}+\vec{n}} .
$$

For irrational $\Lambda, \Lambda^{\prime} \in\left(0, \frac{1}{4}\right)$ one can prove [24] that $\mathscr{L}_{\Lambda}$ and $\mathscr{L}_{\Lambda^{\prime} \neq \Lambda}$ are nonisomorphic. Note that (2.17) and (2.18) are invariant under the transformation

$$
\begin{array}{ll}
T_{\tilde{m}}^{\prime}= \pm T_{A \bar{m}} & A \in G L(2, \mathbb{Z}) \\
& (\operatorname{det} A= \pm 1)
\end{array}
$$

For rational $\Lambda=\frac{M}{N} \mathscr{L}_{\Lambda}$ contains a large ideal (of finite codimension)

$$
I_{\frac{M}{N}}=\left\{\sum_{i} \sum_{\vec{m}} c_{m}^{i}\left(T_{\vec{m}}+N\left(p_{m}^{i}, q_{m}^{i}\right)-T_{\vec{m}}\right) \mid c_{\vec{m}}^{i} \in \mathbb{C}, p_{\vec{m}}^{i}, q_{\vec{m}}^{i} \in \mathbb{Z}\right\} .
$$

For $N$ odd, $M, N$ relative prime integers, dividing out the ideal yields $g l(N, \mathbb{C})$ :

$$
\mathscr{L}_{\Lambda} / I_{\frac{M}{N}} \simeq g l(N, \mathbb{C})
$$

The corresponding basis of $g l(N, \mathbb{C})$ is given by

$$
T_{\bar{m}}=\frac{i N}{4 \pi M} \omega^{\frac{m_{1} m_{2}}{2}} g^{m_{1}} h^{m_{2}}, \quad m_{1}, m_{2}=-\frac{N-1}{2}, \ldots,+\frac{N-1}{2},
$$

where

$$
g=\left(\begin{array}{lllll}
1 & & & & \\
& \omega & & 0 & \\
& & \omega^{2} & & \\
& 0 & & \ddots & \\
& & & & \omega^{N-1}
\end{array}\right), h=\left(\begin{array}{llll}
0 & 1 & & \\
& \ddots & \ddots & \\
& & \ddots & 1 \\
1 & & & 0
\end{array}\right), \quad \omega=e^{4 \pi i \frac{M}{N}}
$$

with

$$
g^{N}=h^{N}=\mathbf{1}, \quad h g=\omega g h .
$$


In all three cases $((2.17),(2.18),(2.22))$ there is an additional one-dimensional ideal generated by $T_{\overrightarrow{0}}$ which, depending on the situation, we will automatically consider as factored out, yielding $\operatorname{sl}(N, \mathbb{C})$ in the case of $(2.22)$, or a certain "dense" subalgebra of the complexified Lie algebra of deformed symplectic diffeomorphisms of the torus, $\operatorname{diff}_{A} T^{2} \simeq \mathscr{L}_{\Lambda}$, in the case of (2.17). Note that for the representation (2.22) one has

$$
T_{\vec{m}}^{\dagger}=-T_{-\vec{m}}, \quad T_{\vec{m}}^{*}=-T_{-m_{1}, m_{2}},
$$

so that

$$
\begin{aligned}
& s l(N, \mathbb{R}) \leftrightarrow x_{\dot{m}}^{*}=-x_{-m_{1}, m_{2}}, \\
& s u(N, \mathbb{R}) \leftrightarrow x_{m}^{*}=x_{-\vec{m}}, \\
& s o(N, \mathbb{R}) \leftrightarrow x_{m}^{*}=x_{-\vec{m}}=-x_{-m_{1}, m_{2}},
\end{aligned}
$$

when writing an element $X$ of $g l(N, \mathbb{C})$ as

$$
X=\sum_{\vec{m}} x_{\vec{m}} T_{\vec{m}}
$$

Both $\mathscr{L}_{\Lambda}$ and $\mathscr{L}_{0}$ permit nontrivial central extensions $\hat{\mathscr{L}}_{\Lambda}$ and $\hat{\mathscr{L}}_{0}[23,25,22]$ by means of a vector $\vec{a}=\left(a_{1}, a_{2}\right) \in \mathbb{C}^{2}$. Let $\hat{\mathscr{L}}_{\Lambda}=\mathscr{L}_{\Lambda} \oplus \mathbb{C} \mathbf{c}$, where $\mathbf{c}$ is the central element. Then

$$
\begin{aligned}
{\left[T_{\vec{m}}, T_{\vec{n}}\right] } & =\frac{1}{2 \pi \Lambda} \sin (2 \pi \Lambda(\vec{m} \times \vec{n})) T_{\vec{m}+\vec{n}}+\vec{a} \cdot \vec{m} \delta_{\vec{m}+\vec{n}, \overrightarrow{0}} \mathbf{c}, \\
{\left[T_{\vec{m}}, \mathbf{c}\right] } & =[\mathbf{c}, \mathbf{c}]=0,
\end{aligned}
$$

which is also defined for $\Lambda \rightarrow 0$. In the star product representation this reads in compact form

$$
[f(\vec{\varphi}), g(\vec{\varphi})]=\{f, g\}_{\star}+\mathbf{c} \int \vec{a} \cdot(\nabla f) g \frac{d \vec{\varphi}}{(2 \pi)^{2}},
$$

where $\{f, g\}_{*}=f * g-g * f$. In the vertex representation we find for the choice $\vec{a}=(a, 0)$

$$
\begin{aligned}
{[F(\theta, T), G(\theta, T)]=} & {[F(\theta, T), G(\theta, T)]_{\mathscr{L}_{A}}+a \operatorname{Tr}\left(G \partial_{\theta} F\right) \mathbf{c} } \\
= & \sum_{m, n}\left(f_{m}(\theta) g_{n}(\theta+\lambda m)-f_{m}(\theta+\lambda n) g_{n}(\theta)\right) T^{m+n} \\
& +a \operatorname{Tr}\left(G \partial_{\theta} F\right) \mathbf{c},
\end{aligned}
$$

where in the second line we have represented $F$ and $G$ as in (2.8).

We close this section by pointing out the relation of $\mathscr{A}_{A}$ to the algebra of magnetic translations in two dimensions, relevant in the discussion of the quantum Hall effect (cf. e.g. [26]). Magnetic translations are generated by the mechanical momentum $\left(p_{i}-\frac{e}{c} A_{i}\right)$, where $p_{i}=\frac{\hbar}{i} \partial_{i}$ is the canonical momentum and $A_{i}$ the vector potential. The generators of finite translations $T_{\vec{\xi}}=e^{\vec{\xi} \cdot\left(\vec{\nabla}-\frac{i e}{\hbar c} \vec{A}\right)}$ satisfy $T_{\vec{\xi}_{1}} T_{\vec{\xi}_{2}}=e^{-i \pi_{\phi}\left(\vec{\xi}_{1} \times \vec{\xi}_{2}\right)} T_{\vec{\xi}_{1}+\vec{\xi}_{2}}$, where $B=\vec{\nabla} \times \vec{A}$ is a uniform perpendicular magnetic 
field and $\phi=\frac{h c}{e}$ the flux quantum. For $\vec{\xi}_{1}$ and $\vec{\xi}_{2}$ linearly independent, $T_{\vec{\xi}_{1}}$ and $T_{\xi_{2}}$ generate a two-dimensional lattice and one finds that for "rational" values of the $B$-field there are closed paths for which the accumulated phase under magnetic translations vanishes.

2.2. Automorphisms and Gradings of $\mathscr{L}_{\Lambda}$. One of the most apparent features of the Lie algebras $\mathscr{L}_{A}$ (as well as their underlying associative algebras, (2.6), which are projective representations of the abelian group $\mathbb{Z}^{2}[22,12]$ ) is their $\mathbb{Z} \times \mathbb{Z}$ grading. In their embedding into $g l_{\infty},(2.3)$, or, when partially completing in one direction (e.g. with respect to $m_{1}$ ), one of the $\mathbb{Z}$-gradings is hidden, while the other is stressed (in (2.3) the grading with respect to the non-zero off-diagonal is manifest, while in the representation (2.2) we have - cf. (2.8) - $\left.\left[f_{m} T^{m}, g_{n} T^{n}\right]=h_{m+n} T^{m+n}\right)$.

For some of the dynamical systems constructed in Chapter three, $\mathbb{Z}_{2}$ gradings of $\mathscr{L}_{A}$ will be essential. With this motivation we will discuss them in some detail. They naturally result from involutive automorphisms of $\mathscr{L}_{A}$. So let

$$
\sigma: \mathscr{L}_{\Lambda} \rightarrow \mathscr{L}_{\Lambda}
$$

have the properties

$$
\sigma^{2}=\mathrm{id}, \quad[\sigma(x), \sigma(y)]=\sigma([x, y]) .
$$

Then $\mathscr{L}_{\Lambda}=\mathscr{L}_{\Lambda}^{+} \oplus \mathscr{L}_{A}^{-}$with

$$
\begin{gathered}
\sigma\left(\mathscr{L}_{A}^{ \pm}\right)= \pm \mathscr{L}_{A}^{ \pm}, \quad\left[\mathscr{L}_{A}^{+}, \mathscr{L}_{A}^{+}\right] \subset \mathscr{L}_{A}^{+}, \\
{\left[\mathscr{L}_{A}^{+}, \mathscr{L}_{A}^{-}\right] \subset \mathscr{L}_{A}^{-}, \quad\left[\mathscr{L}_{A}^{-}, \mathscr{L}_{A}^{-}\right] \subset \mathscr{L}_{A}^{+} .}
\end{gathered}
$$

Observing (2.19), it immediately follows that there exist involutive automorphisms of $\mathscr{L}_{\Lambda}$,

$$
\sigma\left(T_{\vec{m}}\right):= \pm T_{A \vec{m}} \quad \begin{gathered}
A \in G L(2, \mathbb{Z}) \\
A^{2}=1
\end{gathered},
$$

where the $\pm \operatorname{sign}$ in (2.33) corresponds to having $\operatorname{det} A= \pm 1$.

$A$ and $B$ define equivalent automorphisms iff $A$ and $B$ are conjugate to each other in $G L(2, \mathbb{Z})$ and one would like to know a complete set of non-conjugate matrices $A \in G L(2, \mathbb{Z}), A^{2}=\mathbf{1}$. It is given by [27]

$$
\left(\begin{array}{ll}
1 & 0 \\
0 & 1
\end{array}\right),\left(\begin{array}{rr}
-1 & 0 \\
0 & +1
\end{array}\right),\left(\begin{array}{rr}
-1 & 0 \\
0 & -1
\end{array}\right),\left(\begin{array}{ll}
0 & 1 \\
1 & 0
\end{array}\right) \text {. }
$$

The corresponding involutive automorphisms will be referred to as $\hat{\sigma}_{0}, \hat{\sigma}_{1}, \hat{\sigma}_{2}, \hat{\sigma}_{3}$ respectively. In particular

$$
\begin{aligned}
& \hat{\sigma}_{1}\left(T_{\vec{m}}\right):=-T_{-m_{1}, m_{2}}, \\
& \hat{\sigma}_{2}\left(T_{\vec{m}}\right):=+T_{-\vec{m}} .
\end{aligned}
$$

Comparing with (2.24), one finds that for the finite-dimensional case, $\hat{\sigma}_{1}$ corresponds to complex conjugation, and $\hat{\sigma}_{2}$ to minus hermitian conjugation. This also holds for the infinite dimensional representations (2.2) and (2.3), as those also satisfy (2.24). As we defined $\mathscr{L}_{A}$ (as well as $g l(N)$ ) as Lie algebras over $\mathbb{C}$, it is of course necessary to supplement (2.35) by a prescription of how to act on the 
complex-valued coefficients. We need

$$
\begin{aligned}
& \sigma_{1}\left(\sum_{\vec{m}} c_{\vec{m}} T_{\vec{m}}\right):=\sum_{\vec{m}} c_{\vec{m}}^{*} \hat{\sigma}_{1}\left(T_{\vec{m}}\right)=-\sum_{\vec{m}} c_{\vec{m}}^{*} T_{-m_{1}, \boldsymbol{m}_{2}}, \\
& \sigma_{2}\left(\sum_{\vec{m}} c_{\vec{m}} T_{\vec{m}}\right):=\sum_{\vec{m}} c_{\vec{m}}^{*} \hat{\sigma}_{2}\left(T_{\vec{m}}\right)=\sum_{\vec{m}} c_{\vec{m}}^{*} T_{-\vec{m}},
\end{aligned}
$$

in order to justly call $\sigma_{1}$ and $\sigma_{2}$ complex conjugation and minus hermitian conjugation, respectively. Care is needed in the representation (2.4), where $\sigma_{1}$ corresponds to complex conjugation accompanied by $\varphi_{2} \rightarrow-\varphi_{2}$.

When writing elements $F=\sum_{\vec{m}} f_{\vec{m}} T_{\vec{m}}$ (in the representation (2.2)) in the form

$$
F=\sum_{m \geqq 0} f_{m}(\theta) T^{m}+\sum_{m>0} T^{-m} f_{-m}(\theta), \quad T=e^{\lambda \partial_{\theta}},
$$

one can easily check, using

$$
f_{m}(\theta)=\frac{i}{\lambda} \sum_{n} f_{n, m} \omega^{\frac{1}{2}|m| n} e^{i n \theta}
$$

that $\sigma_{1}$ and $\sigma_{2}$ indeed act properly:

$$
\begin{aligned}
& \sigma_{1}(F)=F^{*}=\sum_{m \geqq 0} f_{m}^{*}(\theta) T^{m}+\sum_{m>0} T^{-m} f_{-m}^{*}(\theta), \\
& \sigma_{2}(F)=-F^{\dagger}=-\sum_{m \geqq 0} T^{-m} f_{m}^{*}(\theta)-\sum_{m>0} f_{-m}^{*}(\theta) T^{m} .
\end{aligned}
$$

We will denote the corresponding invariant subalgebras of $\mathscr{L}_{A}$ by $\mathscr{L}_{\Lambda}^{R}$ and $\mathscr{L}_{A}^{U}(R$ standing for "real" and $U$ for unitary, meaning antihermitian for the generators of the algebra):

$$
\sigma_{1}(x)=x, \quad \forall x \in \mathscr{L}_{\Lambda}^{R}, \quad \sigma_{2}(x)=x, \quad \forall x \in \mathscr{L}_{\Lambda}^{U} .
$$

We also define $\mathscr{L}_{A}^{S o}=\left\{x \mid \sigma_{1}(x)=\sigma_{2}(x)=x\right\}$. Subalgebras of this type have previously been considered in [13,28, 12 and 29]. There exist, of course, many other involutive automorphisms, such as

$$
\begin{gathered}
\sigma\left(c_{\vec{m}} T_{\vec{m}}\right)=(-)^{\gamma_{\dot{m}}} c_{\vec{m}}^{*} T_{-\vec{m}}, \\
(-)^{\gamma_{\dot{m}}+\gamma_{\dot{n}}}=(-)^{\gamma_{\dot{m}+\dot{n}}} \quad \forall \vec{m}, \vec{n} \in \mathbb{Z}^{2} .
\end{gathered}
$$

Finally, it seems worth mentioning yet another subalgebra of $\mathscr{L}_{\boldsymbol{A}}$ which, in the vertex representation, is obtained by demanding

$$
f_{ \pm m}(\theta)=0 \quad \text { at } \frac{\theta}{\lambda}=-1,-2, \ldots,-m \quad(m>0)
$$

in (2.37). In order to check that this is consistent with the Lie structure (and the associative structure) one uses (2.9).

2.3. Coadjoint Action and Orbits. Let us introduce the dual space $\hat{\mathscr{L}}_{\Lambda}^{*}$ and the coadjoint action.

In the star-product representation $\mathscr{L}_{A}^{*}$ is the space of linear functionals on the space of trigonometric polynomials $\mathscr{L}_{\boldsymbol{A}}=\{f(\phi)\}$ or other smooth functions on the 
torus. We can define them as integrals using the formalism of distributions

$$
\mathscr{L}_{A}^{*}=\{g \mid\langle g, f\rangle \text { finite }\} \quad \forall f \in \mathscr{L}_{\Lambda},
$$

where we have defined the pairing

$$
\langle g, f\rangle=\operatorname{Tr}\left(g^{*} f\right) .
$$

$\mathscr{L}_{A}^{*}$ contains singular functions as the delta-function or its derivatives and therefore $\mathscr{L}_{\Lambda} \subset \mathscr{L}_{\Lambda}^{*}$.

In the vertex representation $\mathscr{L}_{A}^{*}$ is the space of functionals on a sequence $\left(f_{m}\right)$, where $f_{m}=f_{m}(\theta)$ is a trigonometric polynomial or a smooth function on the circle:

$$
\mathscr{L}_{A}^{*}=\left\{\left(g_{m}\right) \mid \sum_{m \in \mathbb{Z}} \frac{1}{2 \pi} \int_{0}^{2 \pi} d \theta f_{m} g_{m}^{*} \text { finite }\right\} .
$$

Note that if we write $F \in \mathscr{L}_{\Lambda}$ as in (2.8) and likewise for $G \in \mathscr{L}_{\Lambda}^{*}$, the dual space is defined via the pairing $\langle G, F\rangle=\operatorname{Tr}\left(F G^{\dagger}\right)$. The part of $\hat{\mathscr{L}}_{A}^{*}$ corresponding to the central charge is a one-dimensional space and we can define in the star-product representation, for $(f, c) \in \hat{\mathscr{L}}_{A}$ and $(g, d) \in \hat{\mathscr{L}}_{A}^{*}$,

$$
\langle(g, d),(f, c)\rangle=\operatorname{Tr}\left(g^{*} f\right)+c d^{*},
$$

and likewise in the vertex representation, with $(F, c) \in \hat{\mathscr{L}}_{A}$ and $(G, d) \in \hat{\mathscr{L}}_{A}^{*}$

$$
\langle(G, d),(F, c)\rangle=\operatorname{Tr}\left(F G^{\dagger}\right)+c d^{*} .
$$

The coadjoint action of $f \in \mathscr{L}_{A}$ in the $\star$-product representation is then

$$
\operatorname{ad}_{f}^{*}(g, c)=\left(-\left\{f^{*}, g\right\}_{\star}+c\left(\vec{a}^{*} \cdot \vec{\partial}\right) f^{*}, 0\right) .
$$

If

$$
g=\sum_{\vec{m}} g_{\vec{m}} T_{\vec{m}}, \quad f=\sum_{\vec{n}} f_{\vec{n}} T_{\vec{n}}
$$

with $T_{\bar{m}}$ as given in Eq. (2.4), then

$$
\operatorname{ad}_{f}^{*}(g, c)=\left(\sum_{\vec{m}, \vec{n}} \frac{2}{\lambda} \sin \left(\frac{\lambda}{2}(\vec{m} \times \vec{n})\right) f_{-\vec{m}}^{*} g_{\vec{n}} T_{\vec{m}+\vec{n}}-i c \sum_{\vec{m}}\left(\vec{a}^{*} \cdot \vec{m}\right) f_{-\vec{m}}^{*} T_{\vec{m}}, 0\right) .
$$

In the vertex representation we get for the choice $\vec{a}=(1,0)$ for the coadjoint action

$$
\operatorname{ad}_{F}^{*}(G, c)=\left(\left[F^{\dagger}, G\right]_{\mathscr{L}_{A}}+c \partial_{\theta} F^{\dagger}, 0\right) .
$$

Representing $F$ and $G$ as in (2.8), we obtain

$$
\begin{aligned}
\operatorname{ad}_{F}^{*}(G, c)= & \left(\sum _ { m , n } \left[f_{-m}^{*}(\theta+m \lambda) g_{n}(\theta+m \lambda)\right.\right. \\
& \left.-g_{n}(\theta) f_{-_{m}}^{*}(\theta+(m+n) \lambda)\right] T^{m+n} \\
& \left.+c \sum_{m} \partial_{\theta} f_{-m}^{*}(\theta+m \lambda) T^{m}, 0\right) .
\end{aligned}
$$

The actions in both spaces are well defined because of the definitions of $\mathscr{L}_{\Lambda}$ and $\mathscr{L}_{\Lambda}^{*}$.

Thus the orbits are parametrized by pairs $(g, c)\left(g \in \mathscr{L}_{A}^{*}, c \in \mathbb{R}\right)$, where $g$ is defined up to the "gauge" transformations (2.49)-(2.53). 
If $c=0$, then for a subspace in $\mathscr{L}_{\Lambda}^{*}$ we can define invariant functionals (Casimirs)

$$
I_{n}=\operatorname{Tr}\left(g^{n}\right),
$$

where in the star-product representation the $n^{\text {th }}$ power is with respect to $\star$.

The equations of motion for general rigid tops have the standard form of Hamiltonian equations on coadjoint orbits. The Poisson structure on an orbit is induced by the standard Lie brackets in the space of functions on $\mathscr{G}^{*}$. It takes the form of a coadjoint action

$$
\partial_{t} \ell=a_{\frac{\delta H}{\delta \ell}}^{*} \ell, \quad \ell \in \mathscr{G}^{*}, \quad \frac{\delta H}{\delta \ell}=\operatorname{grad} H \in \mathscr{G} .
$$

To describe integrable cases, or, more precisely, to construct integrals of motion, we use the formalism of Lie-Poisson structures in a similar fashion as it was developed in [17] for finite dimensional algebras.

Let us briefly recall this construction. For a Lie algebra $\mathscr{G}$ consider the twisted loop algebra

$$
\mathscr{C}(\mathscr{G}, \sigma)=\sum_{j} \mathscr{G}_{j} \mu^{j}
$$

where $\sigma$ is an automorphism and $\mathscr{G}_{j}$ are homogeneous subspaces (eigenspaces of $\sigma$ ).

Let

$$
\mathscr{C}_{+}(\mathscr{G}, \sigma)=\sum_{j \geqq 0} \mathscr{G}_{j} \mu^{j}, \quad \mathscr{C}_{-}(\mathscr{G}, \sigma)=\sum_{j<0} \mathscr{G}_{j} \mu^{j}
$$

and $P_{ \pm}$the projection operators onto $\mathscr{C}_{ \pm}$parallel to the complementary subalgebra. The Lie-Poisson structure is $\left(R=P_{+}-P_{-}\right)$

$$
\begin{aligned}
{[\xi, \eta]_{R} } & \equiv \frac{1}{2}[R \xi, \eta]+\frac{1}{2}[\xi, R \eta]=\left[\xi_{+}, \eta_{+}\right]-\left[\xi-, \eta_{-}\right], \\
\xi_{ \pm} & =P_{ \pm} \xi, \quad \eta_{ \pm}=P_{ \pm} \eta .
\end{aligned}
$$

We can continue the pairing from $\mathscr{G}$ to $\mathscr{C}(\mathscr{G}, \sigma)$ :

$$
\langle X, Y\rangle_{\mathscr{Q}(\mathscr{S}, \sigma)}=\operatorname{Res}_{\mu=0} \mu^{-1}\langle X(\mu), Y(\mu)\rangle_{\mathscr{G}} d \mu .
$$

We then have with respect to $(2.57)$,

$$
\begin{gathered}
\mathscr{C}^{*}(\mathscr{G}, \sigma)=\mathscr{C}_{+}^{*}(\mathscr{G}, \sigma)+\mathscr{C}^{*}(\mathscr{G}, \sigma), \\
\mathscr{C}_{+}^{*}(\mathscr{G}, \sigma)=\sum_{j \leqq 0} \mathscr{G}_{j}^{*} \mu^{j} \quad \mathscr{C}_{-}^{*}(\mathscr{G}, \sigma)=\sum_{j>0} \mathscr{G}_{j}^{*} \mu^{j},
\end{gathered}
$$

The bracket (2.58) induces $R$-Poisson brackets on $\mathscr{C} *(\lambda, \sigma)$. Invariant polynomials on $\mathscr{C} *(\mathscr{G}, \sigma)$ are

$$
\varphi_{m, n}^{(k)} \equiv \operatorname{Res}_{\mu=0}\left(\mu^{-n} I_{k}\left(\mu^{m} \ell(\mu)\right)\right) d \mu, \quad m, n \in \mathbb{Z} ;
$$

$\ell(\mu) \in \mathscr{C}^{*}(\mathscr{G}, \sigma)$ and $I_{k}$ is a Casimir on the original algebra $\mathscr{G}^{*}$.

The construction of integrable systems is now based on the following two important facts. (i) The $\varphi_{m, n}$ are in involution with respect to the $R$-bracket on $\mathscr{C}^{*}(\lambda, \sigma)$ and (ii) if $H$ has the form (2.61), then the equation of motion with respect 
to the $R$-bracket has the form

$$
\partial_{t} \ell=\operatorname{ad}_{M}^{*} \ell= \pm \operatorname{ad}_{M}^{*} \ell
$$

where $M=R \frac{\delta H}{2 \delta \ell}, M_{ \pm}= \pm P_{ \pm} \frac{\delta H}{\delta \ell}$ and ad* is the standard coadjoint action. These two statements allow us to construct integrable Hamiltonians.

In the following we will put our equations in the form (2.62). The integrals of motion are defined by (2.61).

\section{Dynamical Systems}

3.1. Toda Chain. We consider the central extension $\mathscr{C}\left(\hat{\mathscr{L}}_{A}\right)$ of the loop algebra with values in $\hat{\mathscr{L}}_{\Lambda}$. Let $u(z) \in \mathscr{C}\left(\hat{\mathscr{L}}_{A}\right)$,

with

$$
u(z): S^{1} \rightarrow \hat{\mathscr{L}}_{A}
$$

$$
\begin{array}{ll}
u(z)=(F(z), c(z)), & F(z) \in \mathscr{L}_{\boldsymbol{A}}, \\
& c(z) \text { central charge in } \hat{\mathscr{L}}_{\boldsymbol{A}} .
\end{array}
$$

The commutator of two elements in $\mathscr{C}\left(\hat{\mathscr{L}}_{A}\right)$ is

$$
\left[\left(u(z), k_{1}\right),\left(v(z), k_{2}\right)\right]_{\mathscr{C}\left(\hat{\mathscr{L}}_{A}\right)}=\left([u(z), v(z)]_{\hat{\mathscr{L}}_{A}}, k \int_{S^{1}} d z \operatorname{Tr}_{\mathscr{L}}\left(\partial_{z} u(z) v(z)\right)\right),
$$

where $\operatorname{Tr}_{\mathscr{L}}($,$) is the invariant trace in \mathscr{L}$. In the vertex representation the commutator of two elements $\mathscr{A}, \mathscr{B} \in \mathscr{C}\left(\hat{\mathscr{L}}_{\boldsymbol{A}}\right)$,

is thus

$$
\begin{gathered}
\mathscr{A}=\left(u(z), k_{1}\right)=\left(F(z, \theta, T), c_{1}(z), k_{1}\right), \\
\mathscr{B}=\left(v(z), k_{2}\right)=\left(G(z, \theta, T), c_{2}(z), k_{2}\right)
\end{gathered}
$$

$$
\begin{aligned}
{[\mathscr{A}, \mathscr{B}]_{\mathscr{C}\left(\hat{\mathscr{L}}_{A}\right)}=} & \left([F(z, \theta, T), G(z, \theta, T)]_{\mathscr{L}_{A}}, \int \frac{d T}{T} \frac{d \theta}{2 \pi} G(z, \theta, T) \partial_{\theta} F(z, \theta, T),\right. \\
& \left.\int \frac{d T}{T} \frac{d \theta}{2 \pi} d z G(z, \theta, T) \partial_{z} F(z, \theta, T)\right) .
\end{aligned}
$$

The invariant form on $\mathscr{C}\left(\hat{\mathscr{L}}_{A}\right)$ is

$$
\langle\mathscr{A}, \mathscr{B}\rangle_{\mathscr{C}\left(\hat{\mathscr{L}}_{1}\right)}=\int d z \frac{d T}{T} \frac{d \theta}{2 \pi} F^{\dagger} G+\int d z c_{1}^{*}(z) c_{2}(z)+k_{1}^{*} k_{2}
$$

which allows us to define the dual algebra $\mathscr{C}^{*}\left(\hat{\mathscr{L}}_{A}\right)$. We then find for the coadjoint action on $(\psi(z, \theta, T), c(z), k) \in \mathscr{C} *\left(\hat{\mathscr{L}}_{\Lambda}\right)$ :

$$
\begin{aligned}
\operatorname{ad}_{F(z, \theta, T)}^{*}(\psi(z, \theta, T), c(z), k)= & \left(\left[F^{\dagger}(z, \theta, T), \psi(z, \theta, T)\right] \hat{\mathscr{L}}_{\Lambda}\right. \\
& \left.+\left(c(z) \partial_{\theta}+k \partial_{z}\right) F^{\dagger}(z, \theta, T), 0,0\right) .
\end{aligned}
$$

The Lax pair with spectral parameter $\mu$ and the corresponding equation of motion for the Toda system can now be constructed:

$$
\partial_{t} L=\operatorname{ad}_{M}^{*} L
$$


with

$$
\begin{aligned}
L & =\psi(z, \theta)+\mu T \in \mathscr{C}^{*}\left(\hat{\mathscr{L}}_{A}\right), \\
M^{\dagger} & =\mu^{-1} F(z, \theta) T^{-1} \in \mathscr{C}\left(\hat{\mathscr{L}}_{\Lambda}\right),
\end{aligned}
$$

or

$$
\partial_{t} L-\left(c(z) \partial_{\theta}+k \partial_{z}\right) M^{\dagger}+\left[L, M^{\dagger}\right]=0 .
$$

Separating the $O\left(\mu^{-1}\right)$ and $O\left(\mu^{0}\right)$ terms, we get the equations

$$
\begin{aligned}
\left(c(z) \partial_{\theta}+k \partial_{z}\right) F(z, \theta) & =(\psi(z, \theta)-\psi(z, \theta-\lambda)) F(z, \theta), \\
\partial_{t} \psi(z, \theta) & =F(z, \theta)-F(z, \theta+\lambda) .
\end{aligned}
$$

The first equation is solved by

$$
\begin{aligned}
& F(z, \theta)=e^{\varphi(z, \theta)-\varphi(z, \theta-\lambda)}, \\
& \psi(z, \theta)=\left(c(z) \partial_{\theta}+k \partial_{z}\right) \varphi(z, \theta),
\end{aligned}
$$

and the latter leads to the most general equation of Toda type:

$$
\partial_{t}\left(c(z) \partial_{\theta}+k \partial_{z}\right) \varphi(z, \theta)=e^{\varphi(z, \theta)-\varphi(z, \theta-\lambda)}-e^{\varphi(z, \theta+\lambda)-\varphi(z, \theta)} .
$$

This equation has two regimes. For $k=0$ it is the non-local two-dimensional Toda system first described in [5] where it was derived via reduction and a dressing procedure was proposed, thus proving its integrability. In the approach taken here, the non-locality is due to the central charge of $\hat{\mathscr{L}}_{\Lambda}$. For $k \neq 0$ one is lead to the local two-dimensional Toda system (cf. e.g. $[30,6]$ ). Indeed, if we perform the change of variable with non-singular Jacobian

$$
\begin{aligned}
& \vartheta=\int_{0}^{z} c\left(z^{\prime}\right) d z^{\prime}-k \theta, \\
& z=z,
\end{aligned}
$$

and define $\tilde{\varphi}(z, \vartheta)=\varphi(z, \theta)$, Eq. (3.10) becomes

$$
k \partial_{t} \partial_{z} \tilde{\varphi}(z, \vartheta)=e^{\tilde{\varphi}(z, \vartheta)-\tilde{\varphi}(z, \vartheta+k \lambda)}-e^{\tilde{\varphi}(z, \vartheta-k \lambda)-\tilde{\varphi}(z, \vartheta)} .
$$

This is equivalent to Eq. (3.10) with $c(z)=0$ which is local. Therefore, as long as the central charge $k$ of the loop algebra $\mathscr{C}\left(\hat{\mathscr{L}}_{\Lambda}\right)$ is non-vanishing, the central charge of $\hat{\mathscr{L}}_{\Lambda}$ is irrelevant for the Toda system.

Letting $\varphi_{m}=\varphi(-\lambda m), m \in \mathbb{Z}$, we see that (3.10) (with $c(z)=0, k=1$ ) implies the set of coupled equations

$$
\partial_{t} \partial_{z} \varphi_{m}=e^{\varphi_{m}-\varphi_{m}+1}-e^{\varphi_{m}-1-\varphi_{m}}, \quad m \in \mathbb{Z},
$$

which is the first equation in the Ueno-Takasaki hierarchy $[4,31] .{ }^{2}$ However, whereas in [4] the dependence on $m$ is completely arbitrary, the solutions of (3.13) are strongly constrained if they are to originate from a smooth periodic function (satisfying (3.10)).

Maybe a general remark about $\mathscr{L}_{A}$ and our representations is in place here. We have always used $T$ as $e^{\lambda \partial \theta}$ and an acting on the linear space $V_{1}$ of trigonometric

${ }^{2}$ It is straightforward to generalize our Lax pair along the lines of ref. [4] to generate the whole hierarchy 
polynomials or the space $\hat{V}_{1}$ of smooth (real analytic) functions. As the basic relation

$$
T f(\theta)=f(\theta+\lambda) T
$$

can be realized between integral operators (acting on a much larger space $V \supset \hat{V}_{1} \supset V_{1}$, including distributions) with kernels

$$
\begin{aligned}
& T\left(\theta, \theta^{\prime}\right)=\delta\left(\theta+\lambda-\theta^{\prime}\right), \\
& f\left(\theta, \theta^{\prime}\right)=f(\theta) \delta\left(\theta-\theta^{\prime}\right),
\end{aligned}
$$

one could relax the smoothness condition in (3.10) (thus changing the constraints on the solutions of (3.13) as well). $T$ and $f(\theta)$, when acting on the vectorspace $V_{2} \subset V$, defined as the linear span of $E_{m}=\delta(\theta+\lambda m), m \in \mathbb{Z}$, take the matrix form

$$
(T)_{i j}=\delta_{i, j-1} \quad(f(\theta))_{i j}=f(-j \lambda) \delta_{i j},
$$

which makes contact with the matrix representation (2.3). With this choice, the Lax operators coincide with the ones in [4]. On $V_{1}, T$ is diagonal and $(f(\theta))$ offdiagonal (yet commuting with any other $(g(\theta))$, as it should).

Let us now make some conjectural remarks concerning the local Toda system. Whereas the non-local Toda system was just integrable, the local case seems to be conformal as it is a $N \rightarrow \infty$ limit of the non-periodic $g l_{N}$ Toda system which is conformal. Equation (3.10) (with $c(z)=0$ and $k=1$ ) can be derived from the action

$$
S=\int d \theta \int d t d z\left(\frac{1}{2} \partial_{t} \varphi \hat{\partial}_{z} \varphi+e^{\varphi(\theta-\lambda)-\varphi(\theta)}\right)
$$

or, Eq. (3.13) from the action

$$
S=\int d z d t\left(\frac{1}{2} \partial_{t} \vec{\varphi} \cdot \partial_{z} \vec{\varphi}+\sum_{m} e^{\vec{\alpha}_{m} \cdot \vec{\varphi}}\right),
$$

where $\vec{\varphi}=\left\{\varphi_{m}\right\}$ and $\vec{\alpha}_{m}=(\ldots 0,1,-1,0, \ldots)$ with non-vanishing entries only in the $m^{\text {th }}$ and $(m+1)^{\text {st }}$ positions. Expressions (3.17) and (3.18) resemble the Liouville action. The energy-momentum tensor is traceless and the non-vanishing components are $T_{t t}$ and $T_{z z}$ with

$$
T_{\alpha \alpha}=\frac{1}{2} \partial_{\alpha} \vec{\varphi} \cdot \partial_{\alpha} \vec{\varphi}-\sum_{m} \vec{\gamma}_{m} \cdot \partial_{\alpha}^{2} \vec{\varphi},
$$

where $\vec{\gamma}_{m}=(\ldots, 1,1,0,0, \ldots)$; the change occurs after the $m^{\text {th }}$ entry. In the continuum picture one finds

$$
T_{\alpha \alpha}=\frac{1}{2} \int_{0}^{2 \pi} \frac{d \theta}{2 \pi} \partial_{\alpha} \varphi(\theta) \hat{\partial}_{\alpha} \varphi(\theta)+\frac{1}{\lambda} \int \frac{d \theta}{2 \pi} \theta \partial_{\alpha}^{2} \varphi(\theta) .
$$

In analogy with the $W_{N}$ symmetry of the $g l_{N}$ Toda system, we expect $W(\infty)$ symmetry for the system discussed here and that it describes $W(\infty)$ gravity.

The arguments given above only hold for $\Lambda=\frac{\lambda}{4 \pi}$ irrational. For $2 \Lambda=\frac{M}{N}$ ( $M, N$ relative prime), $\varphi_{N}=\varphi_{0}$, i.e. only $N$ fields couple and we are lead to the periodic Toda chain, which is integrable, but not conformal. For example, if $2 \Lambda=\frac{1}{2}$, for each $\theta \in[0,2 \pi]$ only two fields couple, namely $\varphi_{1}=\varphi(\theta)$ and $\varphi_{2}=\varphi(\theta+\pi)$ and we arrive at the sinh-Gordon equation. 
3.2. Euler Top. Consider a subalgebra $\mathscr{G} \subseteq \mathscr{L}_{\Lambda}$. Let $\ell \in \mathscr{G}^{*}$ and fix a linear operator $\mathscr{A}: \mathscr{G} \rightarrow \mathscr{G}^{*}$. In what follows $\ell$ is called angular momentum, $\mathscr{A}$ is an inertia tensor and $\omega=\mathscr{A}^{-1} \ell \in \mathscr{G}$ is an angular velocity. The generalized Euler top on $\mathscr{G}$ is defined by the equation [19]

$$
\partial_{t} \ell=\operatorname{ad}_{\omega(\ell)}^{*} \ell
$$

This equation is Hamiltonian with respect to

$$
H=\frac{1}{2}\langle\omega(\ell), \ell\rangle=\frac{1}{2}\left\langle\mathscr{A}^{-1} \ell, \ell\right\rangle=\frac{1}{2}\langle\omega, \mathscr{A} \omega\rangle .
$$

The phase space of this system is an orbit of the coadjoint action which is fixed by the Casimirs. In particular, if the central charge $c$ is equal to zero, they have the form (2.54). For $\mathscr{G}=s o(3),(3.21),(3.22)$ is the usual Euler top and the three-wave interaction equation for $S O(3) \mathrm{Kac}-\mathrm{Moody}$ with $c \neq 0$ [32].

In the star-product representation, in accordance with (2.49), Eq. (3.21) takes the form (for $\omega=\omega(\vec{\varphi})$ and $\mathscr{A} \omega(\vec{\varphi})=\ell(\vec{\varphi}) \in \mathscr{G}^{*}$ )

$$
\partial_{t}(\mathscr{A} \omega(\vec{\varphi}))=-\left\{\omega^{*}(\vec{\varphi}), \ell(\vec{\varphi})\right\}_{*}+c(\vec{a} \cdot \vec{\partial}) \omega^{*}(\vec{\varphi}) .
$$

Let $\mathscr{G}$ be the algebra of real functions on $T^{2}$. In the limit $\lambda \rightarrow 0$ algebra $\mathscr{G}$ is isomorphic to the algebra of non-constant symplectic diffeomorphisms of the torus and the star-bracket reduces to the standard Poisson bracket. If we take

$$
\mathscr{A}=\partial_{\varphi_{1}}^{2}+\partial_{\varphi_{2}}^{2}=\Delta
$$

and $c=0$, Eq. (3.23) is the Euler equation of two-dimensional hydrodynamics $[33,19]$

$$
\partial_{t} \Delta \omega=-\left(\partial_{\varphi_{1}} \omega \partial_{\varphi_{2}} \Delta \omega-\partial_{\varphi_{1}} \Delta \omega \partial_{\varphi_{2}} \omega\right) .
$$

Here $\omega$ plays the role of the stream function which defines the velocity field

$$
v_{\varphi_{1}}=-\partial_{\varphi_{2}} \omega, \quad v_{\varphi_{2}}=+\partial_{\varphi_{1}} \omega \text {. }
$$

Therefore, Eq. (3.23) can be considered as a quantum version of two-dimensional hydrodynamics on a torus with $\lambda$ playing the role of Planck's constant [35].

In Fourier modes Eq. (3.23) takes, in correspondence with (2.51), the form $\left(\ell=\sum \ell_{\vec{m}} T_{\vec{m}}, \omega=\sum \omega_{\vec{m}} T_{\vec{m}}\right.$, with the reality condition $\left.\omega_{\vec{m}}=-\omega_{-\vec{m}}^{*}\right)$

$$
\partial_{t} \ell_{\vec{m}}=\frac{2}{\lambda} \sum_{\vec{n}} \omega_{\vec{n}} \ell_{\vec{m}-\vec{n}} \sin \left(\frac{\lambda}{2}(\vec{m} \times \vec{n})\right)+i c(\vec{a} \cdot \vec{m}) \omega_{\vec{m}} .
$$

Note that for $\lambda \rightarrow 0$,

$$
\partial_{t} \ell_{\vec{m}}=\sum_{\vec{n}} \omega_{\vec{n}} \ell_{\vec{m}-\vec{n}}(\vec{m} \times \vec{n})+i c(\vec{a} \cdot \vec{m}) \omega_{\vec{m}}
$$

For the hydrodynamical case (3.25) one gets in this limit

$$
\partial_{t} \omega_{\vec{m}}=\frac{1}{\vec{m}^{2}} \sum_{\vec{n}}(\vec{m}-\vec{n})^{2}(\vec{m} \times \vec{n}) \omega_{\vec{m}-\vec{n}} \omega_{\vec{n}} .
$$

The role of the central charge $c$ in two-dimensional hydrodynamics was discussed in [34]. It takes into account the effect of the overall rotation of the system. 
Let

$$
\mathscr{G}=\mathscr{K}+\mathscr{P}, \quad[\mathscr{K}, \mathscr{K}] \subset \mathscr{K}, \quad[\mathscr{K}, \mathscr{P}] \subset \mathscr{P}, \quad[\mathscr{P}, \mathscr{P}] \subset \mathscr{K}
$$

correspond to the $\mathbb{Z}_{2}$ gradation of some subalgebra $\mathscr{G} \subset \mathscr{L}_{\Lambda}$. In accordance with the definition of the dual space

$$
\mathscr{G}^{*}=\mathscr{K}^{*}+\mathscr{P}^{*}
$$

and in accordance with (3.30) we have

$$
\operatorname{ad}_{\mathscr{K}}^{*} \mathscr{K}^{*} \subset \mathscr{K}^{*}, \quad \operatorname{ad}_{\mathscr{X}}^{*} \mathscr{P}^{*} \subset \mathscr{P}^{*}, \quad \operatorname{ad}_{\mathscr{P}}^{*} \mathscr{K}^{*} \subset \mathscr{P}^{*}, \quad \operatorname{ad}_{\mathscr{P}}^{*} \mathscr{P}^{*} \subset \mathscr{K}^{*} .
$$

We investigate integrable cases of Eq. (3.21). To this end we put

$$
\ell \in \mathscr{K}^{*}, \omega(\ell) \in \mathscr{K} .
$$

Consider the Lax representation with spectral parameter $\mu$ which corresponds to the $\mathbb{Z}_{2}$ gradation (3.30), (3.32),

$$
\begin{aligned}
L=a \mu+\ell \in \mathscr{G}^{*}, & a \in \mathscr{P}^{*}, \\
M=b \mu+\omega \in \mathscr{G}, & b \in \mathscr{P} .
\end{aligned}
$$

The Lax equation

$$
\partial_{t} L=\operatorname{ad}_{M}^{*} L
$$

leads to the equations

$$
\begin{aligned}
\operatorname{ad}_{b}^{*} a & =0, \\
\partial_{t} a & =\operatorname{ad}_{\omega}^{*} a+\operatorname{ad}_{b}^{*} \ell, \\
\partial_{t} \ell & =\operatorname{ad}_{\omega}^{*} \ell .
\end{aligned}
$$

The third equation is the Euler equation (3.21). The first two allow for the description of integrable inertia tensors.

Consider in detail when $\mathscr{G} \simeq \mathscr{G}_{1}=\mathscr{H}+\mathscr{P}$ with $\mathscr{G}_{1} \simeq \mathscr{L}_{\boldsymbol{A}}^{\mathbb{R}}$ and $\mathscr{K} \simeq \mathscr{L}_{\Lambda}^{S O}$. In the vertex representation

$$
\begin{aligned}
& \ell=\sum_{m>0}\left(\ell_{m}(\theta) T^{m}-T^{-m} \ell_{m}(\theta)\right)=-\ell^{\dagger} \in \mathscr{K}^{*}, \\
& \omega=\sum_{m>0}\left(\omega_{m}(\theta) T^{m}-T^{-m} \omega_{m}(\theta)\right)=-\omega^{\dagger} \in \mathscr{K} .
\end{aligned}
$$

From the first equation in (3.36) it follows that we can choose

$$
a=a(\theta)=a^{\dagger}(\theta) \in \mathscr{P}^{*}, \quad b=b(\theta)=b^{\dagger}(\theta) \in \mathscr{P} .
$$

We can also include a central charge in which case $\operatorname{ad}_{M}^{*}=\left[M^{\dagger},\right]+c \partial_{\theta} M^{\dagger}$. Then the second equation in (3.36) becomes

$$
\partial_{t} a(\theta)-c \partial_{\theta}(b(\theta)-\omega(\theta))=[a(\theta), \omega]+[b(\theta), \ell] .
$$

Consider the simplest case, $c=0$, and let $\partial_{t} a=0$. From (3.39) and (3.37) we obtain

$$
\ell_{m}(\theta)=\psi_{m}(\theta) \omega_{m}(\theta)
$$


where

$$
\psi_{m}(\theta)=-\frac{a(\theta)-a(\theta+m \lambda)}{b(\theta)-b(\theta+m \lambda)} .
$$

The functions $\psi_{m}(\theta)$ describe an integrable inertia tensor. They lead to the Hamiltonian

$$
H=-\sum_{m>0} \frac{1}{2 \pi} \int_{0}^{2 \pi} \psi_{m}(\theta) \omega_{m}^{2}(\theta) d \theta .
$$

The energy is positive definite if $\psi_{m}(\theta)>0$. This is, for example, the case if $a(\theta)=1 /(b(\theta))^{r}$ and $b(\theta)>0$. Then, e.g. for $r=1$,

$$
\psi_{m}(\theta)=-\frac{1}{b(\theta) b(\theta+m \lambda)} .
$$

The equation of motion is

$$
\begin{aligned}
\partial_{t} \ell_{m}(\theta)= & \sum_{n>0}\left[\ell_{n}(\theta+\lambda m) \omega_{m+n}(\theta)-\ell_{n}(\theta-\lambda n) \omega_{m+n}(\theta-\lambda n)\right] \\
& +\sum_{n>m}\left[\ell_{n}(\theta-\lambda(n-m)) \omega_{n-m}(\theta-\lambda(n-m))-\ell_{n}(\theta) \omega_{n-m}(\theta+\lambda m)\right] \\
& +\sum_{0<n<m}\left[\ell_{n}(\theta) \omega_{m-n}(\theta+\lambda n)-\ell_{n}(\theta+\lambda(m-n)) \omega_{m-n}(\theta)\right] .
\end{aligned}
$$

To include the dynamics with respect to $\theta$ it is necessary to take $c \neq 0$. Due to (3.39) it leads to a dynamical tensor of inertia. Equation (3.43) acquires the additional term $c \partial_{\theta} \omega_{k}(\theta, t)$ on the right-hand side.

Let us now consider (3.36) in the star-product representation. Here we take $\mathscr{G} \cong \mathscr{G}_{2}=\mathscr{K}+\mathscr{P}$ with $\mathscr{G}=\mathscr{L}_{A}^{u}$ and $\mathscr{K}=\mathscr{L}_{A}^{S O} \cdot \mathscr{L}_{A}^{U}$ consists of purely imaginary functions of $\vec{\varphi}$, while elements of $\mathscr{L}_{A}^{S O}$ have, in addition, to be antisymmetric under $\varphi_{2} \rightarrow-\varphi_{2}$. To satisfy $(3.36)_{1}$ we may choose $(\alpha, \beta \in \mathbb{R})$,

$$
\begin{aligned}
& a=i \alpha\left(\varphi_{1}\right), \\
& b=i \beta\left(\varphi_{1}\right) .
\end{aligned}
$$

Using

and

$$
\begin{aligned}
& e^{i m \varphi_{2}} \star g\left(\varphi_{1}\right)=e^{i m \varphi_{2}} g\left(\varphi_{1}+\frac{\lambda m}{2}\right), \\
& g\left(\varphi_{1}\right) \star e^{i m \varphi_{2}}=e^{i m \varphi_{2}} g\left(\varphi_{1}-\frac{\lambda m}{2}\right),
\end{aligned}
$$

$$
\begin{aligned}
& \ell=i \sum_{m>0} \ell_{m}\left(\varphi_{1}\right) \sin \left(m \varphi_{2}\right) \quad\left(\ell_{m} \in \mathbb{R}\right) \in \mathscr{K}^{*}, \\
& \omega=i \sum_{m>0} \omega_{m}\left(\varphi_{1}\right) \sin \left(m \varphi_{2}\right) \quad\left(\omega_{m} \in \mathbb{R}\right) \in \mathscr{K},
\end{aligned}
$$

$(3.36)_{2}$ yields, with the additional choice $\partial_{t} a=0$,

$$
\ell_{m}\left(\varphi_{1}\right)=\phi_{m}\left(\varphi_{1}\right) \omega_{m}\left(\varphi_{1}\right)
$$


with

$$
\phi_{m}\left(\varphi_{1}\right)=\frac{\alpha\left(\varphi_{1}-\frac{\lambda m}{2}\right)-\alpha\left(\varphi_{1}+\frac{\lambda m}{2}\right)}{\beta\left(\varphi_{1}-\frac{\lambda m}{2}\right)-\beta\left(\varphi_{1}+\frac{\lambda m}{2}\right)} \in \mathbb{R} .
$$

Using this one finds that the Hamiltonian

$$
H=\frac{1}{4} \sum_{m>0} \int_{0}^{2 \pi} \frac{d \varphi}{2 \pi} \phi_{m}(\varphi)\left|\omega_{m}\right|^{2}
$$

is positive for $\phi_{m}>0$. The equation of motion is

$$
\begin{aligned}
\partial_{t} \ell_{m}\left(\varphi_{1}\right)= & \frac{1}{2} \sum_{n>0}\left[\omega_{n}\left(\varphi_{1}+\frac{\lambda}{2}(n+m)\right) \ell_{m+n}\left(\varphi_{1}+\frac{\lambda}{2} n\right)\right. \\
& \left.-\omega_{n}\left(\varphi_{1}-\frac{\lambda}{2}(n+m)\right) \ell_{m+n}\left(\varphi_{1}-\frac{\lambda}{2} n\right)\right] \\
& +\frac{1}{2} \sum_{0<n<m}\left[\omega_{n}\left(\varphi_{1}-\frac{\lambda}{2}(m-n)\right) \ell_{m-n}\left(\varphi_{1}+\frac{\lambda}{2} n\right)\right. \\
& \left.-\omega_{n}\left(\varphi_{1}+\frac{\lambda}{2}(m-n)\right) \ell_{m-n}\left(\varphi_{1}-\frac{\lambda}{2} n\right)\right] \\
& +\frac{1}{2} \sum_{n>m}\left[\omega_{n}\left(\varphi_{1}-\frac{\lambda}{2}(n-m)\right) \ell_{n-m}\left(\varphi_{1}-\frac{\lambda}{2} n\right)\right. \\
& \left.-\omega_{n}\left(\varphi_{1}+\frac{\lambda}{2}(n-m)\right) \ell_{n-m}\left(\varphi_{1}+\frac{\lambda}{2} n\right)\right] .
\end{aligned}
$$

Here we have considered the case of vanishing central charge. A central charge is easily incorporated; we refer to the comments made above in the context of the vertex representation.

3.3. Rigid Body in an External Field. Let us now look at the situation where the decomposition (3.30) $\mathscr{G}=\mathscr{K}+\mathscr{P}$ is also a semidirect sum decomposition. We then have

$$
[\mathscr{K}, \mathscr{K}] \subset \mathscr{K}, \quad[\mathscr{K}, \mathscr{P}] \subset \mathscr{P}, \quad[\mathscr{P}, \mathscr{P}]=0 ;
$$

$\mathscr{G}^{*}=\mathscr{K}^{*}+\mathscr{P}^{*}$ and accordingly

$$
\operatorname{ad}_{\mathscr{K}}^{*} \mathscr{K}^{*} \subset \mathscr{K}^{*}, \quad \operatorname{ad}_{\mathscr{K}}^{*} \mathscr{P}^{*} \subset \mathscr{P}^{*}, \quad \operatorname{ad}_{\mathscr{P}}^{*} \mathscr{K}^{*}=0, \quad \operatorname{ad}_{\mathscr{P}}^{*} \mathscr{P}^{*} \subset \mathscr{K}^{*} .
$$

Let, as previously, $\ell \in \mathscr{K}^{*}$. We introduce the new dynamical field $h \in \mathscr{P}^{*}$. The equations of motion reflecting the structure (3.50) have the so-called "Kirchhoff form"

$$
\begin{aligned}
& \partial_{t} \ell=\operatorname{ad}_{\frac{\delta H}{\delta \ell}}^{*} \ell+\operatorname{ad}_{\frac{\delta H}{\delta h}}^{*} h, \\
& \partial_{t} h=\operatorname{ad}_{\frac{\delta H}{\delta \ell}}^{*} h .
\end{aligned}
$$


For finite dimensional algebras there are many applicable Hamiltonian equations (3.52). Among them are tops in external fields or rigid bodies in an ideal fluid. In many cases these mechanical systems are integrable (see, e.g. the reviews $[20,17]$ ). We shall consider some particular Hamiltonians which are generalizations of (3.22).

Let $\mathscr{C}$ be an operator $\mathscr{C}: \mathscr{P}^{*} \rightarrow \mathscr{P}$ and

$$
H=\frac{1}{2}\langle\omega, \ell\rangle+\frac{1}{2}\langle\mathscr{C} h, h\rangle
$$

If $\mathscr{G}=\mathscr{E}, \mathscr{K}=\operatorname{so}(3), \mathscr{P}=\mathbb{R}^{3}$ and $\mathscr{C}=\operatorname{diag}\left(c_{1}, c_{2}, c_{3}\right)$, this Hamiltonian describes the Clebsch system, namely the motion of a rigid body in an ideal fluid, which is completely integrable for certain values of $c_{i}$. In this case $\omega$ and $\ell$ have the same meaning as for the Euler top and $h \in \mathbb{R}^{3}$ is the momentum of the body-fluid system in a coordinate system rigidly attached to the body.

Let us also consider one of the $\mathbb{Z}_{2}$ graded algebras from Sect. 2.3. The equations of motion in the first representation are generalizations of (3.23). For $h=h(\vec{\varphi})$ and, as previously, $\omega=\omega(\vec{\varphi}), \ell=\mathscr{A} \omega(\vec{\varphi})$, we have

$$
\begin{aligned}
& \partial_{t} \ell=-\left\{\omega^{*}, \ell\right\}_{*}-\left\{(\mathscr{C} h)^{*}, h\right\}_{*}+c(\vec{a} \cdot \vec{\partial})\left(\omega^{*}+(\mathscr{C} h)^{*}\right), \\
& \partial_{t} h=-\left\{\omega^{*}, h\right\}_{*}+c \vec{a} \cdot \vec{\partial} \omega^{*} .
\end{aligned}
$$

(Note that $\mathscr{C}$ can in principle depend on $\vec{\varphi}$.) In Fourier modes this takes the form (for $\ell=\sum \ell_{\vec{m}} T_{\vec{m}}, \omega=\sum \omega_{\vec{m}} T_{\vec{m}}, h=\sum h_{\vec{m}} T_{\vec{m}}, \mathscr{C} h=\sum s_{\vec{m}} T_{\vec{m}}$ ), with $\ell, \omega, h, \mathscr{C} h$ real,

$$
\begin{aligned}
& \partial_{t} \ell_{\vec{m}}=\frac{2}{\lambda} \sum_{\vec{n}}\left(\omega_{\vec{n}} \ell_{\vec{m}-\vec{n}}+s_{\vec{n}} h_{\vec{m}-\vec{n}}\right) \sin \left(\frac{\lambda}{2}(\vec{m} \times \vec{n})\right)+i c(\vec{a} \cdot \vec{m})\left(\omega_{\vec{m}}+s_{\vec{m}}\right), \\
& \partial_{t} h_{\vec{m}}=\frac{2}{\lambda} \sum_{\vec{n}} \omega_{\vec{n}} h_{\vec{m}-\vec{n}} \sin \left(\frac{\lambda}{2}(\vec{m} \times \vec{n})\right)+i c(\vec{a} \cdot \vec{m}) \omega_{\vec{m}} .
\end{aligned}
$$

For $\lambda \rightarrow 0, c=0$ Eqs. (3.54), (3.55) coincide with the equations of magnetohydrodynamics on $T^{2}$ for an ideal fluid with very high conductivity [36]. In this case $\mathscr{A}=$ curl and $\mathscr{C}=\operatorname{curl}$ and $H(\vec{\varphi})$ corresponds to the component of the magnetic field normal to $T^{2}$.

Let us now consider integrable examples. Let

$$
H=\frac{1}{2}\langle\omega, \ell\rangle+\langle b, h\rangle,
$$

where $h \in \mathscr{P}^{*}$ is a dynamical field whereas $b$ is non-dynamical. Then the equations of motion take the form

$$
\begin{aligned}
& \partial_{t} \ell=\operatorname{ad}_{\omega}^{*} \ell+\operatorname{ad}_{b}^{*} h, \\
& \partial_{t} h=\operatorname{ad}_{\omega}^{*} h .
\end{aligned}
$$

In the finite-dimensional situation they correspond to a top in a gravitational field. Following the general recipe we introduce the Lax pair

$$
\begin{aligned}
& L=a \mu+\ell+h \mu^{-1} \subset \mathscr{G}^{*}(\mu, \sigma), \\
& M=b \mu+\omega \subset \mathscr{G}(\mu, \sigma) .
\end{aligned}
$$


The Lax equations lead to the following system

$$
\begin{aligned}
\operatorname{ad}_{b}^{*} a & =0, \\
\partial_{t} a & =\operatorname{ad}_{\omega}^{*} a+\operatorname{ad}_{b}^{*} \ell, \\
\partial_{t} \ell & =\operatorname{ad}_{\omega}^{*} \ell+\operatorname{ad}_{b}^{*} h, \\
\partial_{t} h & =\operatorname{ad}_{\omega}^{*} h .
\end{aligned}
$$

The first two equations coincide with the constraints for the Euler top (first two equations in (3.36)). We now write down Eqs. (3.57) in the vertex representation for $\mathscr{G}_{1}=\mathscr{L}_{A}^{\mathbb{R}} \oplus \mathbf{R}$ which generalize (3.43). For arbitrary $a, b$ which satisfy $(3.59)_{1,2}$, $a=a(\theta, t), b=b(\theta)$,

$$
\begin{aligned}
& \ell=\sum_{m>0}\left[\ell_{m}(\theta) T^{m}-T^{-m} \ell_{m}(\theta)\right]+c \mathbf{c}, \\
& \omega=\sum_{m>0}\left[\omega_{m}(\theta) T^{m}-T^{-m} \omega_{m}(\theta)\right], \\
& h=\sum_{m>0}\left[h_{m}(\theta) T^{m}+T^{-m} h_{m}(\theta)\right]+h_{0}(\theta),
\end{aligned}
$$

and

$$
\begin{aligned}
\partial_{t} \ell_{m}= & \sum_{n>0}\left[\ell_{n}(\theta+\lambda m) \omega_{m+n}(\theta)-\ell_{n}(\theta-\lambda n) \omega_{m+n}(\theta-\lambda n)\right] \\
& +\sum_{n>m}\left[\ell_{n}(\theta-\lambda(n-m)) \omega_{n-m}(\theta-\lambda(n-m))-\ell_{n}(\theta) \omega_{n-m}(\theta+\lambda m)\right] \\
& +\sum_{n<m}\left[\ell_{n}(\theta) \omega_{m-n}(\theta+\lambda n)-\ell_{n}(\theta+\lambda(m-n)) \omega_{n-m}(\theta)\right]+c \partial_{\theta} \omega_{m}(\theta) \\
& +[b(\theta)-b(\theta+\lambda m)] h_{m}(\theta) \\
\partial_{t} h_{m}= & -\sum_{n>0}\left[h_{n}(\theta+\lambda m) \omega_{m+n}(\theta)-h_{n}(\theta-\lambda n) \omega_{m+n}(\theta-\lambda n)\right] \\
& +\sum_{n>m}\left[h_{n}(\theta-\lambda(n-m)) \omega_{n-m}(\theta-\lambda(n-m))-h_{n}(\theta) \omega_{n-m}(\theta+\lambda m)\right] \\
& +\sum_{n<m}\left[h_{n}(\theta) \omega_{m-n}(\theta+\lambda n)-h_{n}(\theta+\lambda(m-n)) \omega_{m-n}(\theta)\right]+c \partial_{\theta} \omega_{m}(\theta) .
\end{aligned}
$$

Acknowledgements. We would like to thank M. Bordemann, D. Lebedev, O. Ogievetski, A. Orlov, M. Schlichenmaier, K. Selivanov and W. Soergel for useful discussions.

\section{References}

1. Drinfel'd, V.G., Sokolov, V.V.: J. Sov. Math. 30, 1975 (1985)

2. Jimbo, M., Miwa, T.: Publ. RIMS 19, 943 (1983)

3. Segal, G., Wilson, G.: Publ. IHES 61, 5 (1985)

4. Ueno, K., Takasaki, K.: Advanced Studies in Pure Mathematics 4, 1 (1984)

5. Degasperis, A., Lebedev, D., Olshanetsky, M., Pakuliak, S., Perelomov, A., Santini, P.: Commun. Math. Phys. 141, 141 (1991)

6. Saveliev, M., Vershik, A.: Commun. Math. Phys. 126, 367 (1989); Phys. Lett 143A, 121 (1990)

7. Bordemann, M., Hoppe, J., Theisen, S.: Phys. Lett. 267B, 374 (1991)

8. Gervais, J.L.: Phys. Lett. 160B, 277 (1985) 
9. Gorsky, A., Olshanetsky, M., Selivanov, K.: On a multiorbit geometrical action for the integrable systems; preprint IC/90/342

10. Fairlie, D., Fletcher, P., Zachos, C.: Phys. Lett. 218B, 203 (1989)

11. Connes, A.: Publ. Math. IHES 62, 257 (1985)

12. Hoppe, J.: Int. J. Mod. Phys. A19, 5235 (1989)

13. Fairlie, D., Fletcher, P., Zachos, C.: J. Math. Phys. 31, 1088 (1990)

14. Floratos, E.G., Illiopoulos, J., Tiktopoulos, G.: Phys. Lett. 217B, 285 (1989)

15. Hoppe, J.: Quantum Theorie of a Massless Relativistic Surface, MIT Ph.D. Thesis, 1982, Part. Res. J. (Kyoto) Vol. 80, 3 (1989/90); Phys. Lett. B250, 44 (1990)

16. Gerasimov, A., Lebedev, D., Morozov, A.: Int. J. Mod. Phys. A6, 977 (1991)

17. Reyman, A.G., Semenov-Tian-Shansky, M.A.: Group Theoretical Methods of Finite Dimensional Integrable Systems

18. Golenischeva-Kutuzova, M.I., Reiman, A.G.: Zap. Nauchn. Sem. 1679, 44 (1988)

19. Arnold, V.A.: Mathematical Methods of Classical Mechanics, Berlin, Heidelberg, New York: Springer 1978

20. Dubrovin, B.A., Krichever, I.M., Novikov, S.P.: In: Encyclopaedia of Mathematical Sciences, Vol 4. Berlin, Heidelberg, New York: Springer 1990.

21. Segal, G.: Int. J. Mod. Phys. A 2859 (1991)

22. Hoppe, J.: Phys. Lett. 215B, 706 (1988)

23. Floratos, E.G.: Phys. Lett. 232B, 467 (1989)

24. Hoppe, J., Schaller, P.: Phys. Lett. 237B, 407 (1990)

25. Floratos, E.G., Iliopoulos, J.: Phys. Lett. 201B, 237 (1988)

26. Bellissard, J.: In: Dorlas T.G. et al. (eds.) Statistical Mechanics and Field Theory: Mathematical Aspects. p. 99, Springer Lecture Notes in Physics, Vol. 257

27. Soergel, W.: private communication

28. Pope, C.N., Romans, L.J.: Class. Quantum Grav. 7, 97 (1990)

29. Golenishcheva-Kutuzova, M., Lebedev, D.: Commun. Math. Phys. 148, 403 (1992)

30. Toda, M.: Theory of Nonlinear Lattices. 2nd ed. Berlin, Heidelberg, New York: Springer 1989

31. Mikhailov, A.V.: Physica 3D, 73 (1981)

32. Zakharov, V.E., Manakov, S.V.: JETP 42, 842 (1976)

33. Landau, L.D., Lifshitz, E.M.: Fluid Mechanics. London: Pergamon Press 1959

34. Zeitlin, V.: Algebraization of 2-d ideal fluid hydrodynamical systems and their finite-mode approximations. preprint

35. Hoppe, J.: (for a 3-dimensional generalisation $(c=0)$ ) Lectures on Integrable Systems. Lecture Notes in Physics, Springer 1992

36. Landau, L.D., Lifshitz, E.M.: Electrodynamics of Continuous Media. London: Pergamon Press 1960

Communicated by J. Fröhlich 